\title{
THz-Wave Generation via Cascaded Optical Parametric Amplification
}

\author{
M. Hemmer ${ }^{1}$, G. Cirmi ${ }^{1,2}$, F. Reichert ${ }^{3}$, K. Ravi ${ }^{1,4}$, F. Ahr ${ }^{1,3}$, H. Çankaya ${ }^{1,2}$, N. H. Matlis ${ }^{1}$, \\ O. D. Mücke ${ }^{1,2}$, L. E. Zapata ${ }^{1}$, and F. X. Kärtner ${ }^{1,2,3,4}$ \\ ${ }^{1}$ Center for Free-Electron Laser Science, DESY, Hamburg, Germany \\ ${ }^{2}$ The Hamburg Center for Ultrafast Imaging, Hamburg, Germany \\ ${ }^{3}$ Physics Department, University of Hamburg, Hamburg, Germany \\ ${ }^{4}$ Department of EECS and RLE, Massachusetts Institute of Technology, Cambridge, USA
}

\begin{abstract}
We demonstrate cascaded optical parametric amplification (COPA) as a promising pathway for efficient generation of THz-waves beyond the Manley-Rowe limit. The spectro-temporal characterization of this novel regime of parametric amplification is reported.
\end{abstract}

\section{INTRODUCTION}

$\mathrm{T}$ he $\mathrm{THz}$ frequency range of the electromagnetic spectrum, located at the boundary between the microwave and optical domains, has for a long time been under-explored. Over the past 20 years however imaging and spectroscopy applications have fueled the interest for this spectral region. Recently, THz frequency fields have been identified as ideal candidates for laser-based charged particle acceleration, triggering a demand for high-energy $\mathrm{THz}$ frequency pulses [1].

Coherent $\mathrm{THz}$ frequency pulses are typically obtained via nonlinear frequency down-conversion of near-IR optical pulses. The Manley-Rowe relations however set an upper limit to the conversion efficiency from near-IR to $\mathrm{THz}$ to a fraction of a percent. This intrinsic limitation has been a hindrance for generating mJ-level $\mathrm{THz}$ frequency pulses as it sets requirements on the driving laser beyond the state of the art.

Here, we report on our numerical and experimental investigations to circumvent the Manley-Rowe limitation and provide a pathway toward multi-mJ $\mathrm{THz}$ frequency optical pulses. Our approach relies on cascaded optical parametric amplification (COPA), a particular regime of optical parametric amplification that allows in situ OPA multiplexing.

\section{IMPLEMENTATION \& RESULTS}

In this proof-of-principle experiment, the COPA is limited to a single-stage amplifier (Fig. 1). This stage consists of a $2 \mathrm{~cm}$ long magnesium oxide doped, periodically poled lithium niobate crystal (MgO:PPLN) with a poling period of $212 \mu \mathrm{m}$ and a $3 \times 3 \mathrm{~mm}^{2}$ clear aperture. Narrowband $(\Delta \lambda<0.5 \mathrm{~nm})$, temporally stretched $(\tau \sim 300 \mathrm{ps})$ near-IR $\left(\lambda_{\mathrm{p}}=1029.45 \mathrm{~nm}\right)$ pump pulses derived from a home-built cryogenically cooled $\mathrm{Yb}: Y A G$ amplifier are employed to energize the amplifier [2]. These pump pulses are used to amplify 300 ps duration, sub$0.5 \mathrm{~nm}$ bandwidth signal pulses with center wavelength at $\lambda_{\mathrm{s}}=$ $1031.2 \mathrm{~nm}$ in a collinear geometry. The idler wave resulting from the interaction is a multi-cycle $0.5 \mathrm{THz}$ field.

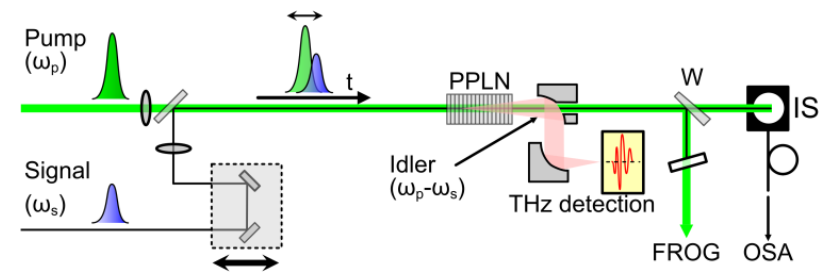

Fig. 1. Layout of the cascaded optical parametric amplifier featuring a $2 \mathrm{~cm}$ long PPLN crystal, spectro-temporal characterization at optical wavelength and $\mathrm{THz}$ yield monitoring - IS: integrating sphere, $\mathrm{W}$ : wedge

We spectrally characterized the evolution of the signal wave in the optical domain as a function of both seed and pump fluence. We find - in agreement with numerical simulations [3] - that increasing the seed pulse energy results in redshifting of the cascaded orders with respect to the pump wavelength a critical feature to achieve THz-wave generation. Our numerical simulations reveal that preferential red-shifting is essential for efficient $\mathrm{THz}$ generation and is driven by the dispersion in the PPLN crystal. Increasing the pump fluence results in an increase in the number of cascaded orders and an increase in THz yield (Fig. 2). Up to 10 spectral lines have been recorded and $\mathrm{THz}$ energies in the one hundred $\mathrm{nJ}$ energy range have been measured. We attribute the modest $\mathrm{THz}$ yield Energy-calibrated spectral measurements revealed a significant $(\sim 50 \%)$ depletion of the pump pulses.

The temporal characterization of both the pump and signal waves in the optical domain - via second harmonic generation frequency resolved optical gating (SHG-FROG) - revealed temporal modulations of the seed signal pulse at $500 \mathrm{GHz}$ repetition rate ( 2 ps period), confirming the presence of phaselocking between the cascaded orders. Beyond efficient $\mathrm{THz}$ generation the cascaded optical parametric amplification scheme could find application in frequency-comb science, delivering record high energy density sparse frequency combs.

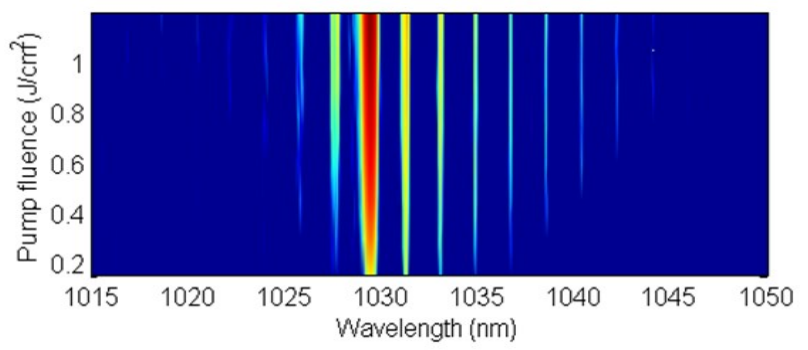

Fig. 2 Measured cascaded spectrum as a function of pump fluence (log scale) showing preferential red-shifting. 


\section{CONCLUSION \& FUTURE WORK}

We have experimentally demonstrated cascaded parametric amplification. Our numerical simulations show good agreement with the experimental results and promise scaling beyond the Manley-Rowe limit, therefore providing a pathway toward multi-mJ $\mathrm{THz}$ frequency pulse generation. Future work includes refinement of the experimental conditions via in particular implementation of cryogenic cooling of the PPLN crystal and multi-stage amplification. Due to the exponential nature of amplification, abatement of absorption by cryogenic cooling potentially offer percent level conversion efficiencies. COPA also find applications for optical frequency combs and $\mathrm{THz}$ wave generation.

\section{REFERENCES}

[1] E. Nanni et al., Nat. Comm.6, 8486 (2015)

[2] M. Hemmer et al., Conference on Laser and Electro-Optics (CLEO), paper Stu4O.3, San Jose, CA (USA), 2015.

[3] K. Ravi et al. Conference on Laser and Electro-Optics (CLEO), paper SM2L.6, San Jose, CA (USA), 2016. 